\title{
Cubatures on Grassmannians: moments, dimension reduction, and related topics
}

\author{
Anna Breger, Martin Ehler, Manuel Gräf, and Thomas Peter
}

\section{Introduction}

Function approximation, integration, and inverse problems are just few examples of numerical fields that rely on efficient strategies for function sampling. As particular sampling rules, the concepts of cubatures in Euclidean space and the sphere have been widely investigated to integrate polynomials by a finite sum of sampling values, cf. [22, 30, 39, 41, 47]. To some extent, cubatures are universal sampling strategies in the sense that they are highly efficient in many fields, and in the context of function approximation, covering, and integration they have proved superior to the widely used random sampling $[12,52]$.

Recently, cubatures on compact manifolds have attracted attention, cf. [11, $32,49]$. Integration, covering, and polynomial approximation from cubatures on manifolds and homogeneous spaces have been extensively studied from a theoretical point of view, cf. [20, 24, 34, 43, 52] and references therein. Orthogonality is a leading concept in many mathematical fields, and dimension reduction is intrinsically tied together with low dimensional projections. The Grassmannian manifold is the space of orthogonal projectors of fixed rank,

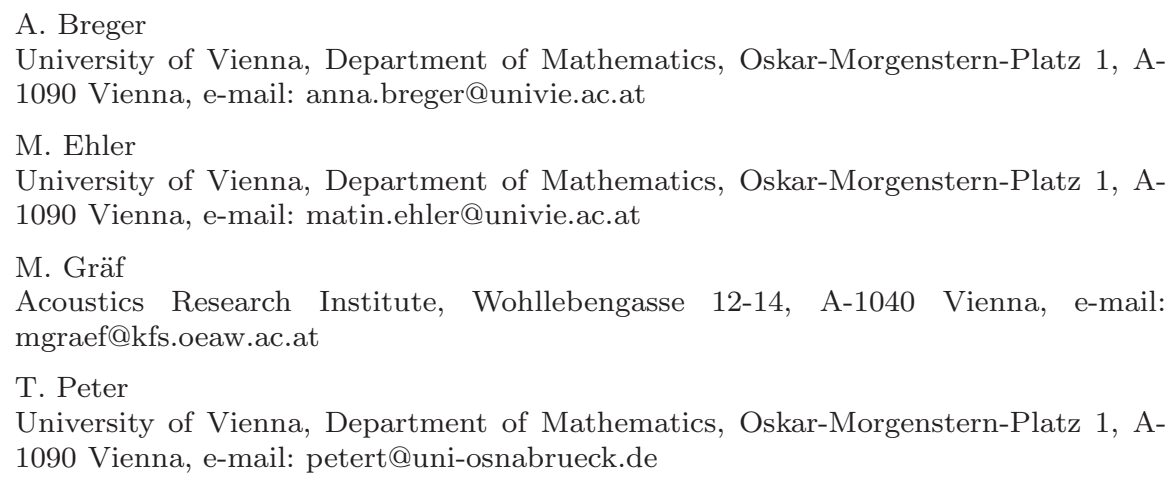


and in this chapter we like to explore on the concept of cubatures in Grassmannians. Therefore, we shall provide a brief overview of recent results on Grassmannian cubatures.

Our starting point in Section 2 is the problem of reconstructing a sparse (i.e. finitely supported) probability measure $\mu$ in $\mathbb{R}^{d}$ from its first few moments. Sparse distributions are indeed uniquely determined by their first few moments, and Prony's method has recently been adapted to this reconstruction, [8, 42]. According to the Johnson Lindenstrauss Lemma, low dimensional projections of $\mu$ still capture essential information, [19]. Taking the first few moments of low dimensional projections only, we now aim to reconstruct the first few moments of $\mu$ but we allow for general probability distributions in Section 3, not necessarily sparse ones, cf. [10]. A new construction of suitable projections are provided in Theorem 1. It turns out that the choice of projectors is closely linked to cubatures in Grassmannians, i.e., the set of low dimensional projectors should form a cubature, see Section 4 and, in particular, Theorem 2. Hence, the reconstruction of high dimensional moments from lower dimensional ones is naturally related to the concept of Grassmannian cubatures. Therefore, we then discuss in Section 5.1 numerical constructions of cubatures in Grassmannians by minimizing the worst case integration error of polynomials, cf. [14, 4]. In Section 5.2, we go beyond polynomials and briefly discuss sequences of low cardinality cubatures that yield optimal worst case integration error rates for Bessel potential functions, cf. [11], see also [14]. The optimal integration errors of cubatures directly induce schemes for function approximation from samples by replacing the inner products of $L_{2}$ orthonormal basis expansions with cubature approximations, see Section 5.3. Intuitively, good samplings for function approximation should well cover the underlying space, and, in Section 5.4, we recapitulate that sequences of low cardinality Grassmannian cubatures are asymptotically optimal coverings, cf. [13]. To further reflect on the versatility, we also provide some results on phase retrieval problems, in which Grassmannian cubatures are used, see Section 5.5 for details.

So far, we have outlined the use of Grassmannian cubatures for various topics in numerical mathematics. Unions of Grassmannians would offer additional flexibility since the rank of the projector is not needed to be fixed a-priori. Projectors with varying ranks may indeed have benefits in practice, see $[38,55]$ for potential applications. Therefore, the concept of cubatures on unions of Grassmannians is discussed in Section 6. The number of required cubature points is mainly steered by the dimension of the underlying polynomial space. Addressing necessary prerequisites for the aforementioned topics within unions of Grassmannians, i.e., approximation of integrals and functions, moment reconstruction, covering, and phase retrieval, we shall determine the dimensions of polynomial spaces on unions of Grassmannians, cf. [28]. For special cases, we provide elementary proofs. The general cases need deeper analysis, for which we refer to [28]. 


\section{Reconstruction from moments and dimension reduction}

\subsection{Reconstructing sparse distributions from moments}

Our starting point is a high dimensional random vector $X \in \mathbb{R}^{d}$ with finite support $\left\{x_{i}\right\}_{i=1}^{m} \subset \mathbb{R}^{d}$, i.e., $X$ is distributed according to a discrete probability measure $\mu$ on $\mathbb{R}^{d}$ with support $\left\{x_{i}\right\}_{i=1}^{m}$ and positive weights $\left\{a_{i}\right\}_{i=1}^{m}$ satisfying $\sum_{i=1}^{m} a_{i}=1$, so that

$$
\mu=\sum_{i=1}^{m} a_{i} \delta_{x_{i}}
$$

where $\delta_{x_{i}}$ denotes the point measure at $x_{i}$. We now aim to reconstruct $\mu$ from knowledge of the moments

$$
m_{\mu}(\lambda):=\mathbb{E} X^{\lambda}=\sum_{i=1}^{m} a_{i} x_{i}^{\lambda}, \quad \lambda \in \Lambda
$$

where $\Lambda \subset \mathbb{N}^{d}$ is some fixed subset. The nonlinear inverse problem of reconstructing $\mu$ means to identify its support $\left\{x_{i}\right\}_{i=1}^{m}$ and its weights $\left\{a_{i}\right\}_{i=1}^{m}$. The core idea of Prony's method is to determine an ideal $\mathcal{I}$ of polynomials on $\mathbb{R}^{d}$ just from the moments $m_{\mu}(\lambda), \lambda \in \Lambda$, through a system of linear equations, such that its zero locus

$$
\mathcal{V}(\mathcal{I})=\left\{x \in \mathbb{R}^{d}: f(x)=0, \forall f \in \mathcal{I}\right\}
$$

is exactly the point set $\left\{x_{i}\right\}_{i=1}^{m}$. The one dimensional case, expressed in terms of difference equations, was introduced in [8], the multivariate case is treated in $[42]$.

Once $\mathcal{I}$ is determined, its zero locus $\mathcal{V}(\mathcal{I})=\left\{x_{i}\right\}_{i=1}^{m}$ can be determined by standard methods [9], and the weights $\left\{a_{i}\right\}_{i=1}^{m}$ are computed by a system of linear equations from the Vandermonde system (1).

More specifically, the zero locus $\mathcal{V}\left(\mathcal{I}_{i}\right)$ of each ideal

$$
\mathcal{I}_{i}:=\left(\left(z-x_{i}\right)^{\alpha}: \alpha \in \mathbb{N}^{d},|\alpha|=1\right)
$$

is $\mathcal{V}\left(\mathcal{I}_{i}\right)=\left\{x_{i}\right\}$, for $i=1, \ldots, m$, so that $\left\{x_{i}\right\}_{i=1}^{m}=\mathcal{V}(\mathcal{I})$ with $\mathcal{I}:=\mathcal{I}_{1} \cdots \mathcal{I}_{m}$. Note that $\mathcal{I}$ coincides with

$$
\mathcal{I}=\left(\prod_{i=1}^{m}\left(z-x_{i}\right)^{\alpha_{i}}: \alpha_{i} \in \mathbb{N}^{d},\left|\alpha_{i}\right|=1, i=1, \ldots, m\right)
$$

so that we have $d^{m}$ many generators of the ideal that must now be determined from the moments $m_{\mu}(\lambda), \lambda \in \Lambda$. 
To simplify, let us now suppose that $d=1$. In this case, the ideal $\mathcal{I}$ is generated by the single polynomial

$$
p(z)=\left(z-x_{1}\right) \cdots\left(z-x_{m}\right)=\sum_{k=0}^{m} p_{k} z^{k}
$$

of degree $m$. Its coefficient sequence $\left\{p_{k}\right\}_{k=0}^{m}$ satisfies

$$
\sum_{k=0}^{m} p_{k} m_{\mu}(k+\lambda)=\sum_{i=1}^{m} x_{i}^{\lambda} a_{i} \sum_{k=0}^{m} p_{k} x_{i}^{k}=\sum_{i=1}^{m} x_{i}^{\lambda} a_{i} p\left(x_{i}\right)=0 .
$$

Equation (2) holds for arbitrary values of $\lambda$. Thus, varying $\lambda$ und using that $p_{m}=1$ leads to the linear system of equations

$$
\sum_{k=0}^{m-1} p_{k} m_{\mu}(k+\lambda)=-m_{\mu}(m+\lambda), \quad \lambda \in \Lambda^{\prime},
$$

where $\Lambda^{\prime} \subset \Lambda$ such that $k+\Lambda^{\prime} \in \Lambda$, for all $k=0, \ldots, m$. We now attempt to solve (3) for $p_{0}, \ldots, p_{m-1}$. Obviously, $\Lambda$ must be sufficiently large, so that

$$
H:=\left(m_{\mu}(k+\lambda)\right) \underset{\substack{\lambda \in \Lambda^{\prime} \\ k=0, \ldots, m-1}}{i, \mathbb{R}^{\left|\Lambda^{\prime}\right| \times m}}
$$

can have full rank $m$. From knowledge of $p$, the eigenvalues of its companion matrix yield its zeros $\left\{x_{i}\right\}_{i=1}^{m}$. Having determined $\left\{x_{i}\right\}_{i=1}^{m}$, (1) yields a Vandermonde system of linear equations to compute the weights $\left\{a_{i}\right\}_{i=1}^{m}$. Note that the rank condition in (4) is satisfied for $\Lambda=\{0, \ldots, 2 m-1\}$ and $\Lambda^{\prime}=\{0, \ldots, m-1\}$, cf. [50] and [51] for an overview of Prony methods.

The case $d>1$ is more involved, but can essentially be treated similarly. In [42] it is shown that $\# \Lambda=\mathcal{O}\left(m^{d}\right)$ suffices to ensure reconstruction while $\# \Lambda=\mathcal{O}(m d)$ suffices if $\left\{x_{i}\right\}_{i=1}^{m}$ are in general position.

Concerning numerical stability, one has to differentiate between the idea of Prony's method as presented here and stable numerical variants for implementation as for example ESPRIT [53], MUSIC [54], and finite rate of innovation [56]. These algorithms perform excellent in applications. If $\lambda$ and $k$ are chosen as proposed in (2), the system matrix (4) is a Hankel matrix that can be factored into

$$
H=A^{\top} D A
$$

with a diagonal matrix $D=\operatorname{diag}\left(a_{i}\right)_{i=1}^{m}$ and a Vandermonde matrix $A=$ $\left(x_{i}^{k}\right)_{k=0, i=1}^{m-1, m}$. For $d>1$, a similar factorization holds, where $A$ is a generalized Vandermonde matrix. Due to the Vandermonde structure of $A$, its condition number tends to be large if the minimal separation distance $\nu_{\mu}$ is small or if there are large sphere deviations $\alpha_{\mu}$, i.e.,

$$
\nu_{\mu}:=\min _{i \neq j}\left\|x_{i}-x_{j}\right\|_{2} \approx 0, \quad \text { or } \quad \alpha_{\mu}:=\max _{i \neq j}\left|\left\|x_{i}\right\|-\left\|x_{j}\right\|\right| \gg 0 .
$$


This pinpoints stable performances when the measure $\mu$ has a well-separated support on a sphere with well behaved weights.

Note that the Prony method works beyond probability measures and can deal with $x_{i} \in \mathbb{C}^{d}, a_{i} \in \mathbb{C}$ and to this end also with $\lambda \in \mathbb{Z}^{d}$. Indeed, if $\Lambda^{\prime}$ in (2) is chosen as $\Lambda^{\prime} \subset-\mathbb{N}^{d}$, then the resulting system matrix becomes a Toeplitz matrix, which is preferred in some literature on Prony's method.

\subsection{Dimension reduction}

The idea of dimension reduction is that properties of interest of the high dimensional random vector $X \in \mathbb{R}^{d}$ may still be captured within its orthogonal $k<d$ dimensional projection, i.e., in $P X$, where $P$ is an element in the Grassmannian space

$$
\mathcal{G}_{k, d}:=\left\{P \in \mathbb{R}_{\text {sym }}^{d \times d}: P^{2}=P ; \operatorname{Tr}(P)=k\right\} .
$$

Here $\mathbb{R}_{\mathrm{sym}}^{d \times d}$ is the set of symmetric matrices in $\mathbb{R}^{d \times d}$. Consider now two sparsely distributed random vectors

$$
X, Y \sim \sum_{i=1}^{m} a_{i} \delta_{x_{i}}
$$

Their difference $X-Y$ is distributed according to

$$
X-Y \sim \sum_{i, j=1}^{m} a_{i} a_{j} \delta_{x_{i}-x_{j}} .
$$

For $P \in \mathcal{G}_{k, d}$, the magnitude of the differences is distributed according to

$$
\|P X-P Y\|^{2} \sim \sum_{i, j=1}^{m} a_{i} a_{j} \delta_{\left\|P x_{i}-P x_{j}\right\|^{2}} .
$$

In fact, for $0<\epsilon<1$ and $k$ with $d \geq k \geq \frac{4 \log (m)}{\epsilon^{2} / 2-\epsilon^{3} / 2}$, there is $P \in \mathcal{G}_{k, d}$, such that

$$
(1-\epsilon)\|X-Y\|^{2} \leq \frac{d}{k}\|P X-P Y\|^{2} \leq(1+\epsilon)\|X-Y\|^{2}
$$

holds with probability 1 . This is the direct consequence of realizations of the Johnson-Lindenstrauss Lemma applied to the deterministic point set $\left\{x_{i}\right\}_{i=1}^{m}$, cf. [19].

Note that (6) tells us that the dimension reduction still preserves essential information of $X$ and $Y$. At this point though, we just know of its existence, and we have not yet specified any particular projector $P$ such that (6) holds, see $[19,1,44]$ for different types of random choices. 
We should point out that $P X$ and $P Y$ are contained in a $k$ dimensional subspace of $\mathbb{R}^{d}$, but still have $d$ entries as vectors in $d$ dimensions. The actual dimension reduction takes place by applying $Q \in \mathcal{V}_{k, d}$ with $Q^{\top} Q=P$, where

$$
\mathcal{V}_{k, d}:=\left\{Q \in \mathbb{R}_{\mathrm{sym}}^{k \times d}: Q Q^{\top}=I_{k}\right\}
$$

denotes the Stiefel manifold. The inequality (6) becomes

$$
(1-\epsilon)\|X-Y\|^{2} \leq \frac{d}{k}\|Q X-Q Y\|^{2} \leq(1+\epsilon)\|X-Y\|^{2},
$$

where $Q X, Q Y \in \mathbb{R}^{k}$ are properly dimension reduced random vectors still containing the information of the pairwise differences up to a factor $1 \pm \epsilon$.

\section{High dimensional moments from lower dimensional ones}

We shall now combine dimension reduction with a modified problem, which is related to the reconstruction from moments. First, we drop the sparsity conditions and allow for arbitrary probability measures $\mu$ on $\mathbb{R}^{d}$. Let $X \in \mathbb{R}^{d}$ be some random vector with unknown Borel probability distribution on $\mathbb{R}^{d}$. Suppose we do not have access to its moments, but we observe the first few moments of order $T$ of low-dimensional linear projections, i.e., for $\left\{Q_{j}\right\}_{j=1}^{n} \subset$ $\mathcal{V}_{k, d}$, we measure

$$
\mathbb{E}\left(Q_{j} X\right)^{s}, \quad s \in \mathbb{N}^{k},|s| \leq T,
$$

We cannot reconstruct $\mu$ directly, but we aim to determine the first few highdimensional moments

$$
\mathbb{E} X^{r}, \quad r \in \mathbb{N}^{d},|r| \leq T .
$$

In other words, we know the first few moments of order $T$ of the dimension reduced random vectors $Q_{j} X \in \mathbb{R}^{k}, j=1, \ldots, n$ and our task is to reconstruct the high-dimensional moments, cf. [10]. The idea is to interpret moments as algebraic polynomials and represent desired high degree polynomials as products of polynomials of lower degree. The remainder of this chapter is dedicated to establish precise relations. by

Polynomials of total degree $T$ on $\mathbb{R}^{d}$, denoted by $\operatorname{Pol}_{T}\left(\mathbb{R}^{d}\right)$, are decomposed

$$
\operatorname{Pol}_{T}\left(\mathbb{R}^{d}\right)=\bigoplus_{t=0}^{T} \operatorname{Hom}_{t}\left(\mathbb{R}^{d}\right),
$$

where $\operatorname{Hom}_{t}\left(\mathbb{R}^{d}\right)$ denotes the space of homogeneous polynomials of degree $t$ on $\mathbb{R}^{d}$. Let $x \in \mathbb{R}^{d}$ be a vector of unknowns, then $\left(Q_{j} x\right)^{s}$ is a homogenous polynomial of degree $|s|$. If 


$$
\left\{\left(Q_{j} x\right)^{s}: j=1, \ldots, n, s \in \mathbb{N}^{k},|s|=t\right\}
$$

spans $\operatorname{Hom}_{t}\left(\mathbb{R}^{d}\right)$, then each monomial of order $t$ is a linear combination of elements in (9), so that the linearity of the expectation yields that all high-dimensional moments of order $t$ can be reconstructed from the lowdimensional moments

$$
\mathbb{E}\left(Q_{j} X\right)^{s}, \quad j=1, \ldots, n, \quad|s|=t .
$$

Thus, we aim to find $\left\{Q_{j}\right\}_{j=1}^{n}$, such that, for each $t \leq T,(9) \operatorname{spans}^{H_{0}}\left(\mathbb{R}^{d}\right)$. Note that spanning sets in finite dimensions are also called frames.

The most excessive dimension reduction corresponds to $k=1$. In this case, we observe that we only need to address $t=T$ :

Proposition 1. Let $\left\{Q_{j}\right\}_{j=1}^{n} \subset \mathcal{V}_{1, d}$ and $x \in \mathbb{R}^{d}$ be a vector of unknowns.

a) If $\left\{\left(Q_{j} x\right)^{t}\right\}_{j=1}^{n}$ is a frame for $\operatorname{Hom}_{t}\left(\mathbb{R}^{d}\right)$, then $\left\{\left(Q_{j} x\right)^{t-1}\right\}_{j=1}^{n}$ is a frame for $\operatorname{Hom}_{t-1}\left(\mathbb{R}^{d}\right)$.

b) If $\left\{\left(Q_{j} x\right)^{t-1}\right\}_{j=1}^{n}$ is linearly independent in $\operatorname{Hom}_{t-1}\left(\mathbb{R}^{d}\right)$, then $\left\{\left(Q_{j} x\right)^{t}\right\}_{j=1}^{n}$ is linearly independent in $\operatorname{Hom}_{t}\left(\mathbb{R}^{d}\right)$.

Proof. a) Let $f$ be an arbitrary element in $\operatorname{Hom}_{t-1}\left(\mathbb{R}^{d}\right)$. There is $g \in$ $\operatorname{Hom}_{t}\left(\mathbb{R}^{d}\right)$ such that its first partial derivative $\partial_{1} g$ coincides with $f$. Since $\left\{\left(Q_{j} x\right)^{t}\right\}_{j=1}^{n}$ is a frame for $\operatorname{Hom}_{t}\left(\mathbb{R}^{d}\right)$, there are coefficients $\left\{c_{j}\right\}_{j=1}^{n}$ such that $g=\sum_{j=1}^{n} c_{j}\left(Q_{j} x\right)^{t}$. Therefore, we obtain

$$
f(x)=\sum_{j=1}^{n} c_{j}\left(Q_{j} e_{1}\right) t\left(Q_{j} x\right)^{t-1}
$$

which verifies part a).

b) Suppose that $0=\sum_{j=1}^{n} c_{j}\left(Q_{j} x\right)^{t}$. Applying all partial derivatives yield

$$
0=\sum_{j=1}^{n} c_{j}\left(Q_{j} e_{i}\right) t\left(Q_{j} x\right)^{t-1}, \quad i=1, \ldots, d
$$

The linear independence assumption implies $c_{j}\left(Q_{j} e_{i}\right)=0$, for $i=1, \ldots, d$, and, therefore, $c_{j}=0$, for $j=1, \ldots, n$, since $Q_{j} \neq 0$.

Part a) of Proposition 1 tells us that if $\left\{\left(Q_{j} x\right)^{t}\right\}_{j=1}^{n}$ is a frame for $\operatorname{Hom}_{t}\left(\mathbb{R}^{d}\right)$, then

$$
\left\{\left(Q_{j} x\right)^{s}: j=1, \ldots, n, s \in \mathbb{N}^{k},|s| \leq t\right\}
$$

is a frame for $\mathrm{Pol}_{t}\left(\mathbb{R}^{d}\right)$. The proof directly shows that the first low-dimensional moments are sufficient to reconstruct the first high-dimensional moments.

Next, we provide a general construction recipe of $\left\{Q_{j}\right\}_{j=1}^{n} \subset \mathcal{V}_{1, d}$ that covers arbitrary $d$ and $t$. Note that the dimension of $\operatorname{Hom}_{t}\left(\mathbb{R}^{d}\right)$ is $\left(\begin{array}{c}t+d-1 \\ d-1\end{array}\right)$ : 
Theorem 1. Let $\left\{v_{i}\right\}_{i=1}^{d}$ be pairwise different positive real numbers, let $\left\{\alpha_{j}\right\}_{j=1}^{t+d-1}$ be pairwise different nonnegative integers, and let $V=\left(v_{i}^{\alpha_{j}}\right)_{i, j}$ denote the associated $(t+d-1) \times d$-Vandermonde type matrix. Suppose that the $\left(\begin{array}{c}t+d-1 \\ d-1\end{array}\right) \times d$ matrix $Q$ is build from all minors of $V$ of order $d-1$. We denote the rows of $Q$ by $Q_{1}, \ldots, Q_{n}$, where $n=\left(\begin{array}{c}t+d-1 \\ d-1\end{array}\right)$. Then $\left\{\left(Q_{j} x\right)^{t}\right\}_{j=1}^{n}$ is a basis for $\operatorname{Hom}_{t}\left(\mathbb{R}^{d}\right)$.

Proof. We expand $\left(Q_{j} x\right)^{t}$ by the multivariate binomial formula

$$
\left(Q_{j} x\right)^{t}=\sum_{\alpha \in \mathbb{N}^{d},|\alpha|=t}\left(\begin{array}{l}
t \\
\alpha
\end{array}\right) Q_{j}^{\alpha} x^{\alpha} .
$$

The coefficients are put into the $j$-th row of a matrix $M_{1} \in \mathbb{R}^{n \times n}$, i.e.,

$$
M_{1}=\left(\left(\begin{array}{c}
t \\
\alpha
\end{array}\right) Q_{j}^{\alpha}\right)_{j, \alpha} .
$$

We must now check that $M_{1}$ is invertible.

Dividing each column $\alpha$ by its respective binomial coefficient $\left(\begin{array}{l}t \\ \alpha\end{array}\right)$ yields the matrix $M_{2}=\left(Q_{j}^{\alpha}\right)_{j, \alpha} \in \mathbb{R}^{n \times n}$, and $M_{1}$ is invertible if and only if $M_{2}$ is. Let $c$ denote the product of all minors of order $d$ of $V$. It follows from [57] that

$$
\operatorname{det}\left(M_{2}\right)=c^{d-1} .
$$

The Vandermonde structure yields that $c \neq 0$, so that $M_{2}$ and hence $M_{1}$ is invertible. Thus, $\left\{\left(Q_{j} x\right)^{t}\right\}_{j=1}^{n}$ is indeed a basis for $\operatorname{Hom}_{t}\left(\mathbb{R}^{d}\right)$.

Note that normalization of the rows of $Q$ in Theorem 1 yields $\left\{Q_{j}\right\}_{j=1}^{n} \subset \mathcal{V}_{1, d}$, and $\left\{\left(Q_{j} x\right)^{t}\right\}_{j=1}^{n}$ is a basis for $\operatorname{Hom}_{t}\left(\mathbb{R}^{d}\right)$. Thus, for each $s \leq t,\left\{\left(Q_{j} x\right)^{s}\right\}_{j=1}^{n}$ is a frame for $\operatorname{Hom}_{s}\left(\mathbb{R}^{d}\right)$ according to Proposition 1.

\section{Frames vs. cubatures and a moment reconstruction formula}

So far, we have seen that reconstruction of high dimension moments from low dimensional ones is related to frames for $\operatorname{Hom}_{t}\left(\mathbb{R}^{d}\right)$. Next, we shall relate such frames to cubature points. Let $\operatorname{Hom}_{t}\left(\mathbb{S}^{d-1}\right)$ denote the space of homogeneous polynomials $\operatorname{Hom}_{t}\left(\mathbb{R}^{d}\right)$, but restricted to the sphere $\mathbb{S}^{d-1}$. For points $\left\{q_{j}\right\}_{j=1}^{n} \subset \mathbb{S}^{d-1}$ and weights $\left\{\omega_{j}\right\}_{j=1}^{n} \subset \mathbb{R}$, we say that $\left\{\left(q_{j}, \omega_{j}\right)\right\}_{j=1}^{n}$ is a cubature for $\operatorname{Hom}_{t}\left(\mathbb{S}^{d-1}\right)$ if

$$
\int_{\mathbb{S}^{d-1}} f(x) \mathrm{d} x=\sum_{j=1}^{n} \omega_{j} f\left(q_{j}\right), \quad \text { for all } f \in \operatorname{Hom}_{t}\left(\mathbb{S}^{d-1}\right),
$$


where $d x$ denotes the standard measure on the sphere normalized to have mass one. It turns out that the frame property of $\left\{\left(Q_{j} x\right)^{s}\right\}_{j=1}^{n}$ is related to the concept of cubature points.

Theorem 2. Let $\left\{Q_{j}\right\}_{j=1}^{n} \subset \mathcal{V}_{1, d}$ and $x \in \mathbb{R}^{d}$ be a vector of unknowns.

a) If $\left\{\left(Q_{j} x\right)^{t}\right\}_{j=1}^{n}$ is a frame for $\operatorname{Hom}_{t}\left(\mathbb{R}^{d}\right)$, then there are weights $\left\{\omega_{j}\right\}_{j=1}^{n} \subset$ $\mathbb{R}$, such that $\left\{\left(Q_{j}^{\top}, \omega_{j}\right)\right\}_{j=1}^{n}$ is a cubature for $\operatorname{Hom}_{t}\left(\mathbb{S}^{d-1}\right)$.

b) If there are weights $\left\{\omega_{j}\right\}_{j=1}^{n} \subset \mathbb{R}$ such that $\left\{\left(Q_{j}^{\top}, \omega_{j}\right)\right\}_{j=1}^{n}$ is a cubature for $\operatorname{Hom}_{2 t}\left(\mathbb{S}^{d-1}\right)$, then $\left\{\left(Q_{j} x\right)^{t}\right\}_{j=1}^{n}$ is a frame for $\operatorname{Hom}_{t}\left(\mathbb{R}^{d}\right)$.

Proof. a) Since $\left\{\left(Q_{j} x\right)^{t}\right\}_{j=1}^{n}$ is a frame for $\operatorname{Hom}_{t}\left(\mathbb{R}^{d}\right)$, for each $a \in \mathbb{S}^{d-1}$, there are coefficients $\left\{c_{j}(a)\right\}_{j=1}^{n} \subset \mathbb{R}$ such that

$$
\left(a^{\top} x\right)^{t}=\sum_{j=1}^{n} c_{j}(a)\left(Q_{j} x\right)^{t} .
$$

Note that the mapping $a \mapsto c_{j}(a)$ can be chosen to be continuous, for each $j=1, \ldots, n$. Therefore, we derive

$$
\int_{\mathbb{S}^{d-1}}\left(a^{\top} x\right)^{t} \mathrm{~d} a=\sum_{j=1}^{n}\left(Q_{j} x\right)^{t} \int_{\mathbb{S}^{d-1}} c_{j}(a) \mathrm{d} a=\sum_{j=1}^{n}\left(Q_{j} x\right)^{t} \omega_{j},
$$

with $\omega_{j}=\int_{\mathbb{S}^{d-1}} c_{j}(a) \mathrm{d} a$. Since the above equality holds for all $x \in \mathbb{R}^{d}$, $\left\{\left(Q_{j}^{\top}, \omega_{j}\right)\right\}_{j=1}^{n}$ is a cubature for $\operatorname{Hom}_{t}\left(\mathbb{S}^{d-1}\right)$.

b) Note that $\operatorname{Hom}_{t}\left(\mathbb{S}^{d-1}\right)$ is a reproducing kernel Hilbert space and let us denote its reproducing kernel with respect to the standard inner product by $K_{t}$. For now, we restrict $x$ to the sphere and let $a \in \mathbb{S}^{d-1}$ as well. The reproducing property yields

$$
\left(a^{\top} x\right)^{t}=\int_{\mathbb{S}^{d-1}}\left(z^{\top} x\right)^{t} K_{t}(z, a) \mathrm{d} z .
$$

The mapping $z \mapsto\left(z^{\top} x\right)^{t} K_{t}(z, a)$ is contained in $\operatorname{Hom}_{2 t}\left(\mathbb{S}^{d-1}\right)$, so that the cubature property yields

$$
\left(a^{\top} x\right)^{t}=\sum_{j=1}^{n} \omega_{j}\left(Q_{j} x\right)^{t} K_{t}\left(Q_{j}^{\top}, a\right)=\sum_{j=1}^{n}\left(Q_{j} x\right)^{t} c_{j}(a),
$$

where $c_{j}(a)=\omega_{j} K_{t}\left(Q_{j}^{\top}, a\right)$. A homogeneity argument concludes the proof.

Note that the degree of the homogeneous polynomials in Part b) of Theorem 2 is not the same but $2 t$ for the cubatures and $t$ for the frame. The degree $2 t$ is due to multiplication of two homogeneous polynomials of degree $t$, which is not just an artifact of the proof. There are indeed cubatures for $\operatorname{Hom}_{t}\left(\mathbb{S}^{d-1}\right)$, whose cardinality is lower than the dimension of $\operatorname{Hom}_{t}\left(\mathbb{R}^{d}\right)$. 
In fact, Theorem 2 holds in much more generality in suitable finite dimensional reproducing kernel Hilbert spaces. Let $(\Omega, \sigma)$ be a finite measure space and let $\mathcal{F}$ be a linear subspace of continuous functions in $L_{2}(\Omega, \sigma)$. For points $\left\{q_{j}\right\}_{j=1}^{n} \subset \Omega$ and weights $\left\{\omega_{j}\right\}_{j=1}^{n} \subset \mathbb{R}$, we say that $\left\{\left(q_{j}, \omega_{j}\right)\right\}_{j=1}^{n}$ is a cubature for $\mathcal{F}$ if

$$
\int_{\Omega} f(x) \mathrm{d} \sigma(x)=\sum_{j=1}^{n} \omega_{j} f\left(q_{j}\right), \quad \text { for all } f \in \mathcal{F} .
$$

The following result generalizes Theorem 2:

Proposition 2. Let $K: \Omega \times \Omega \rightarrow \mathbb{R}$ be a symmetric kernel that linearly generates $\mathcal{F}$, i.e., $K(x, y)=K(y, x)$, for $x, y \in \Omega$, and

$$
\mathcal{F}=\operatorname{span}\{K(a, \cdot): a \in \Omega\} .
$$

For $\left\{q_{j}\right\}_{j=1}^{n} \subset \Omega$, the following holds:

a) If $\left\{K\left(q_{j}, \cdot\right)\right\}_{j=1}^{n}$ is a frame for $\mathcal{F}$, then there are weights $\left\{\omega_{j}\right\}_{j=1}^{n} \subset \mathbb{R}$, such that $\left\{\left(q_{j}, \omega_{j}\right)\right\}_{j=1}^{n}$ is a cubature for $\mathcal{F}$.

b) If there are weights $\left\{\omega_{j}\right\}_{j=1}^{n} \subset \mathbb{R}$ such that $\left\{\left(q_{j}, \omega_{j}\right)\right\}_{j=1}^{n}$ is a cubature for the linear span of $\mathcal{F} \cdot \mathcal{F}$, then $\left\{K\left(q_{j}, \cdot\right)\right\}_{j=1}^{n}$ is a frame for $\mathcal{F}$.

The proof of Proposition 2 is structurally the same as for Theorem 2 with $K(x, y)=\left(x^{\top} y\right)^{t}$ and $\mathcal{F}=\operatorname{Hom}_{t}\left(\mathbb{S}^{d-1}\right)$, so we omit the details.

Remark 1. Part b) of Proposition 2 implies $n \geq \operatorname{dim}(\mathcal{F})$. Analoguous results in [21], for instance, are restricted to positive weights.

Note that $Q \in \mathcal{V}_{1, d}$ if and only if $Q^{\top} Q \in \mathcal{G}_{1, d}$. Moreover, the kernel

$$
K_{t, 1}: \mathcal{G}_{1, d} \times \mathcal{G}_{1, d} \rightarrow \mathbb{R}, \quad(P, R) \mapsto \operatorname{trace}(P R)^{t}
$$

linearly generates $\operatorname{Hom}_{t}\left(\mathcal{G}_{1, d}\right)$. Here, the space of homogeneous polynomials of degree $t$ on $\mathcal{G}_{k, d}$ is defined by restrictions of homogeneous polynomials of degree $t$ on $\mathbb{R}_{\mathrm{sym}}^{d \times d}$. Moreover, $\mathcal{G}_{k, d}$ is naturally endowed with an orthogonal invariant probability measure $\sigma_{k, d}$. For $x \in \mathbb{S}^{d-1}$,

$$
(Q x)^{2 t}=K_{t, 1}\left(Q^{\top} Q, x x^{\top}\right),
$$

so that $\operatorname{Hom}_{2 t}\left(\mathbb{S}^{d-1}\right)$ corresponds to $\operatorname{Hom}_{t}\left(\mathcal{G}_{1, d}\right)$. According to (12) we deduce that, for $\left\{Q_{j}\right\}_{j=1}^{n} \subset \mathcal{V}_{1, d}$, the set $\left\{\left(Q_{j} x\right)^{2 t}\right\}_{j=1}^{n}$ is a frame for $\operatorname{Hom}_{2 t}\left(\mathbb{R}^{d}\right)$ if and only if $\left\{K_{t, 1}\left(Q_{j}^{\top} Q_{j}, \cdot\right)\right\}_{j=1}^{n}$ is a frame for $\operatorname{Hom}_{t}\left(\mathcal{G}_{1, d}\right)$. Similarly, $\left\{\left(Q_{j}^{\top}, \omega_{j}\right)\right\}_{j=1}^{n}$ is a cubature for $\operatorname{Hom}_{2 t}\left(\mathbb{S}^{d-1}\right)$ if and only if $\left\{\left(Q_{j}^{\top} Q_{j}, \omega_{j}\right)\right\}_{j=1}^{n}$ is a cubature for $\operatorname{Hom}_{t}\left(\mathcal{G}_{1, d}\right)$. Therefore, we can switch to the Grassmannian setting to formulate the following moment reconstruction result:

Corollary 1 ([10]). For $r \in \mathbb{N}^{d}$ with $|r| \leq t \leq d$, there are coefficients $a_{s}^{r} \in$ $\mathbb{R}, s \in \mathbb{N}^{d},|s|=|r|$, such that if $\left\{\left(P_{j}, \omega_{j}\right)\right\}_{j=1}^{n}$ is a cubature for $\operatorname{Hom}_{t}\left(\mathcal{G}_{1, d}\right)$, then any random vector $X \in \mathbb{R}^{d}$ satisfies 


$$
\mathbb{E} X^{r}=\sum_{j=1}^{n} \omega_{j} \sum_{s \in \mathbb{N}^{d},|s|=|r|} a_{s}^{r} \mathbb{E}\left(P_{j} X\right)^{s} .
$$

For $Q_{j} \in \mathcal{V}_{1, d}$ and $P_{j} \in \mathcal{G}_{1, d}$ with $P_{j}=Q_{j}^{\top} Q_{j}$, one switches between the moments of $Q_{j} X$ and $P_{j} X$ by the formula

$$
\left(P_{j} X\right)^{s}=Q_{j}^{s}\left(Q_{j} X\right)^{|s|}, \quad s \in \mathbb{N}^{d} .
$$

It may depend on the context when $P_{j} X$ or $Q_{j} X$ is preferred.

\section{Cubatures in Grassmannians}

Proposition 2 connects frames and cubatures beyond $\mathcal{G}_{1, d}$ and applies to the general Grassmannians $\mathcal{G}_{k, d}$ by $\Omega=\mathcal{G}_{k, d}, \mathcal{F}=\operatorname{Hom}_{t}\left(\mathcal{G}_{k, d}\right)$, and the kernel $K=K_{t, k}$ given by

$$
K_{t, k}: \mathcal{G}_{k, d} \times \mathcal{G}_{k, d} \rightarrow \mathbb{R}, \quad(P, R) \mapsto \operatorname{trace}(P R)^{t},
$$

cf. $[10,28]$. In the following sections, we shall provide further examples for the usefulness of Grassmannian cubatures beyond moment reconstruction. To begin with, we address the issue of constructing cubatures.

\subsection{Numerical construction of cubatures}

Cubatures on Grassmannians with constant weights are constructed in [3] from group orbits. There is also a simple method to numerically compute cubature points by some minimization method as we shall outline next. The $t$-fusion frame potential for points $\left\{P_{j}\right\}_{j=1}^{n} \subset \mathcal{G}_{k, d}$ and weights $\left\{\omega_{j}\right\}_{j=1}^{n} \subset \mathbb{R}$ is

$$
\operatorname{FFP}\left(\left\{\left(P_{j}, \omega_{j}\right)\right\}_{j=1}^{n}, t\right):=\sum_{i, j=1}^{n} \omega_{j} \omega_{i} \operatorname{trace}\left(P_{i} P_{j}\right)^{t} .
$$

Assuming that $\sum_{j=1}^{n} \omega_{j}=1$, the fusion frame potential is lower bounded by

$$
\operatorname{FFP}\left(\left\{\left(P_{j}, \omega_{j}\right)\right\}_{j=1}^{n}, t\right) \geq \int_{\mathcal{G}_{k, d}} \int_{\mathcal{G}_{k, d}} \operatorname{trace}(P R)^{t} \mathrm{~d} \sigma_{k, d}(P) \mathrm{d} \sigma_{k, d}(R),
$$

cf. [14] and also [4]. Since the constant functions are contained in $\operatorname{Hom}_{t}\left(\mathcal{G}_{k, d}\right)$, any cubature must satisfy $\sum_{j=1}^{n} \omega_{j}=1$.

Theorem $3([\mathbf{1 4}, \mathbf{4}])$. If $\sum_{j=1}^{n} \omega_{j}=1$ and (14) holds with equality, then $\left\{\left(P_{j}, \omega_{j}\right)\right\}_{j=1}^{n}$ is a cubature for $\operatorname{Hom}_{t}\left(\mathcal{G}_{k, d}\right)$. 
In order to check for equality in (14), we require a more explicit expression for the right-hand-side. In fact, it holds

$$
\int_{\mathcal{G}_{k, d}} \int_{\mathcal{G}_{k, d}} \operatorname{trace}(P R)^{t} \mathrm{~d} \sigma_{k, d}(P) \mathrm{d} \sigma_{k, d}(R)=\sum_{\substack{|\pi|=t, \ell(\pi) \leq d / 2}} \frac{C_{\pi}^{2}\left(I_{k}\right)}{C_{\pi}\left(I_{d}\right)}
$$

where $I_{d}$ denotes the $d \times d$ identity matrix and $\pi$ is an integer partition of $t$ with $\ell(\pi)$ being the number of nonzero parts, and $C_{\pi}$ are the zonal polynomials, cf. $[17,46,37]$. Evaluation of $C_{\pi}$ at $I_{k}$ and $I_{d}$, respectively, yields

$$
C_{\pi}\left(I_{d}\right)=2^{|\pi|}|\pi| !\left(\frac{d}{2}\right)_{\pi} \prod_{1 \leq i<j \leq \ell(\pi)}\left(2 \pi_{i}-2 \pi_{j}-i+j\right) / \prod_{i=1}^{\ell(\pi)}\left(2 \pi_{i}+\ell(\pi)-i\right) !,
$$

cf. [25]. Here, $(a)_{\pi}$ denotes the generalized hypergeometric coefficient given by

$$
(a)_{\pi}:=\prod_{i=1}^{\ell(\pi)}\left(a-\frac{1}{2}(i-1)\right)_{\pi_{i}}, \quad(a)_{s}:=a(a+1) \ldots(a+s-1) .
$$

Fixing the weights $\left\{\omega_{j}\right\}_{j=1}^{n} \subset \mathbb{R}$, say $\omega_{j}=1 / n$, for $j=1, \ldots, n$, we can now aim to numerically minimize the $t$-fusion frame potential $\operatorname{FFP}\left(\left\{\left(P_{j}, \omega_{j}\right)\right\}_{j=1}^{n}, t\right)$ over all sets of $n$ points $\left\{P_{j}\right\}_{j=1}^{n} \subset \mathcal{G}_{k, d}$ and check for equality in (14), where the right-hand-side can be computed explicitly. See $[13,14]$ for successful minimizations in $\mathcal{G}_{2,4}$.

\subsection{Cubatures for approximation of integrals}

Cubature points enable us to replace integrals over polynomials by finite sums. We now aim to go beyond polynomials and keep track of the integration error. Without loss of generality, we assume $k \leq \frac{d}{2}$ throughout since $\mathcal{G}_{d-k, d}$ can be identified with $\mathcal{G}_{k, d}$.

The eigenfunctions $\left\{\varphi_{\pi}\right\}_{\ell(\pi) \leq k}$ of the Laplace-Beltrami operator $\Delta$ on $\mathcal{G}_{k, d}$ are an orthonormal basis for $L_{2}\left(\mathcal{G}_{k, d}\right)$ and are naturally indexed by integer partitions $\pi$ of length at most $k$. Let $\left\{-\lambda_{\pi}\right\}_{\ell(\pi) \leq k}$ be the corresponding eigenvalues, i.e.,

$$
\lambda_{\pi}=2|\pi| d+4 \sum_{i=1}^{k} \pi_{i}\left(\pi_{i}-i\right),
$$

cf. [40, Theorem 13.2]. Without loss of generality, we choose each $\varphi_{\pi}$ to be real-valued, in particular, $\varphi_{(0)} \equiv 1$. Essentially following [11, 45], we formally 
define $(I-\Delta)^{s / 2} f$ to be the distribution on $\mathcal{G}_{k, d}$, such that

$$
\left\langle(I-\Delta)^{s / 2} f, \varphi_{\pi}\right\rangle=\left(1+\lambda_{\pi}\right)^{s / 2}\left\langle f, \varphi_{\pi}\right\rangle, \quad \text { for all } \ell(\pi) \leq k .
$$

The Bessel potential space $H_{p}^{s}\left(\mathcal{G}_{k, d}\right)$, for $1 \leq p \leq \infty$ and $s \geq 0$, is

$$
\begin{aligned}
H_{p}^{s}\left(\mathcal{G}_{k, d}\right) & :=\left\{f \in L_{p}\left(\mathcal{G}_{k, d}\right):\|f\|_{H_{p}^{s}}<\infty\right\}, \quad \text { where } \\
\|f\|_{H_{p}^{s}} & :=\left\|(I-\Delta)^{s / 2} f\right\|_{L_{p}},
\end{aligned}
$$

i.e., $f \in H_{p}^{s}\left(\mathcal{G}_{k, d}\right)$ if and only if $f \in L_{p}\left(\mathcal{G}_{k, d}\right)$ and $(I-\Delta)^{s} f \in L_{p}\left(\mathcal{G}_{k, d}\right)$.

The expected worst case error of integration in Bessel potential spaces of $n$ independent random points endowed with constant weights is of the order $n^{-\frac{1}{2}}$ :

Proposition $3([35,48,12,14])$. For $s>k(d-k) / 2$, suppose $P_{1}, \ldots, P_{n}$ are random points on $\mathcal{G}_{k, d}$, independently identically distributed according to $\sigma_{k, d}$ then it holds

$$
\sqrt{\mathbb{E}\left[\sup _{\substack{f \in H_{2}^{s}\left(\mathcal{G}_{k, d}\right) \\\|f\|_{H_{2}^{s}} \leq 1}}\left|\int_{\mathcal{G}_{k, d}} f(P) \mathrm{d} \sigma_{k, d}(P)-\frac{1}{n} \sum_{j=1}^{n} f\left(P_{j}\right)\right|^{2}\right]}=c n^{-\frac{1}{2}}
$$

with $c^{2}=\sum_{1 \leq \ell(\pi) \leq k}\left(1+\lambda_{\pi}\right)^{-s}$.

The following result follows from [11, Theorem 2.12]:

Theorem 4 ([11]). Let $s>k(d-k) / p$. Any sequence of cubatures $\left\{\left(P_{j}^{(t)}, \omega_{j}^{(t)}\right)\right\}_{j=1}^{n_{t}}$ with nonnegative weights for $\operatorname{Hom}_{t}\left(\mathcal{G}_{k, d}\right), t=1,2, \ldots$, satisfies, for $f \in$ $H_{p}^{s}\left(\mathcal{G}_{k, d}\right)$,

$$
\left|\int_{\mathcal{G}_{k, d}} f(P) \mathrm{d} \sigma_{k, d}(P)-\sum_{j=1}^{n_{t}} \omega_{j}^{t} f\left(P_{j}^{t}\right)\right| \lesssim t^{-s}\|f\|_{H_{p}^{s}}
$$

Remark 2. For any $t=1,2, \ldots$, there exist cubatures $\left\{\left(P_{j}^{(t)}, \omega_{j}^{(t)}\right)\right\}_{j=1}^{n_{t}}$ for $\operatorname{Hom}_{t}\left(\mathcal{G}_{k, d}\right)$ with positive weights such that

$$
n_{t} \asymp t^{k(d-k)}, \quad t=1,2, \ldots,
$$

cf. [21]. Grassmannian $t$-designs are cubatures for $\operatorname{Hom}_{t}\left(\mathcal{G}_{k, d}\right)$ with constant weights $\omega_{j}=1 / n$, for $j=1, \ldots, n$, and there exist Grassmannian $t$-designs that satisfy the asymptotics (17), cf. [31].

If (17) is satisfied, then Theorem 4 leads to

$$
\left|\int_{\mathcal{G}_{k, d}} f(P) \mathrm{d} \mu_{k, d}(P)-\sum_{j=1}^{n_{t}} \omega_{j}^{t} f\left(P_{j}^{t}\right)\right| \lesssim n_{t}^{-\frac{s}{k(d-k)}}\|f\|_{H_{p}^{s}} .
$$


The condition $s>k(d-k) / p$, for $p=2$, in Theorem 17 then yields that cubature points do better than the random points in Proposition 3. Given any sequence of points of cardinality $n_{t}$, the rate $n_{t}^{-\frac{s}{k(d-k)}}$ cannot be improved, cf. [11].

\subsection{Cubatures for function approximation}

The basic idea for applying cubature points in function approximation is quite simple. The standard expansion of any $f \in L_{2}\left(\mathcal{G}_{k, d}\right)$ in the orthogonal basis $\left\{\varphi_{\pi}\right\}_{\ell(\pi) \leq k}$ yields

$$
f=\sum_{\ell(\pi) \leq k}\left\langle f, \varphi_{\pi}\right\rangle \varphi_{\pi} \approx \sum_{\substack{|\pi| \leq t \\ \ell(\pi) \leq k}}\left\langle f, \varphi_{\pi}\right\rangle \varphi_{\pi}
$$

where the approximation is simply derived by truncating the infinite series at $|\pi| \leq t$. The inner product $\left\langle f, \varphi_{\pi}\right\rangle$ is an integral that we approximate by the concept of cubatures, i.e., the error for approximating the integral by a finite sum is steered by (18):

$$
\begin{aligned}
\sum_{\substack{|\pi| \leq t \\
\ell(\pi) \leq k}}\left\langle f, \varphi_{\pi}\right\rangle \varphi_{\pi} & =\sum_{\substack{|\pi| \leq t \\
\ell(\pi) \leq k}} \int_{\mathcal{G}_{k}, d} f(P) \varphi_{i}(P) \mathrm{d} \sigma_{k, d}(P) \varphi_{i} \\
& \approx \sum_{\substack{|\pi| \leq t \\
\ell(\pi) \leq k}} \sum_{j=1}^{n} \omega_{j} f\left(P_{j}\right) \varphi_{\pi}\left(P_{j}\right) \varphi_{\pi} \\
& =\sum_{j=1}^{n} \omega_{j} f\left(P_{j}\right) \sum_{\substack{|\pi| \leq t \\
\ell(\pi) \leq k}} \varphi_{\pi}\left(P_{j}\right) \varphi_{\pi} .
\end{aligned}
$$

If we define $K_{t}(P, R)=\sum_{\substack{|\pi| \leq t \\ \ell(\pi) \leq k}} \varphi_{\pi}(P) \varphi_{\pi}(R)$, then we arrive at the approximation

$$
f \approx \sum_{j=1}^{n} \omega_{j} f\left(P_{j}\right) K_{t}\left(P_{j}, \cdot\right)
$$

The right-hand-side of (21) is composed by two separate approximations, truncation of the series (19) and the approximation of the integral via cubatures (20). To obtain suitable error rates, it turns out that we better replace the sharp truncation by a smoothed version, i.e., we define the kernel $K_{t}$ on $\mathcal{G}_{k, d} \times \mathcal{G}_{k, d}$ by 


$$
K_{t}(P, Q)=\sum_{\ell(\pi) \leq k} h\left(t^{-2} \lambda_{\pi}\right) \varphi_{\pi}(P) \varphi_{\ell}(Q),
$$

where $h: \mathbb{R}_{\geq 0} \rightarrow \mathbb{R}$ is an infinitely often differentiable and nonincreasing function with $h(x)=1$, for $x \leq 1 / 2$, and $h(x)=0$, for $x \geq 1$. The smoothed series truncation becomes the expression

$$
\sigma_{t}(f):=\int_{\mathcal{G}_{k, d}} f(P) K_{t}(P, \cdot) \mathrm{d} \sigma_{k, d}(P),
$$

and $\sigma_{t}(f)$ approximates $f$ with an error rate that matches the ones in Theorem 4 :

Theorem 5 ([45]). If $f \in H_{p}^{s}\left(\mathcal{G}_{k, d}\right)$, then

$$
\left\|f-\sigma_{t}(f)\right\|_{L_{p}} \lesssim t^{-s}\|f\|_{H_{p}^{s}}
$$

To approximate $f$ from finitely many samples, we combine the smoothed truncation with cubature points to replace the integral by a finite sum. For sample points $\left\{P_{j}\right\}_{j=1}^{n} \subset \mathcal{G}_{k, d}$ and weights $\left\{\omega_{j}\right\}_{j=1}^{n}$, we define

$$
\sigma_{t}\left(f,\left\{\left(P_{j}, \omega_{j}\right)\right\}_{j=1}^{n}\right):=\sum_{j=1}^{n} \omega_{j} f\left(P_{j}\right) K_{t}\left(P_{j}, \cdot\right)
$$

which coincides with (21) but the kernel $K_{t}$ is from (22). Note that we must now consider functions $f$ in Bessel potential spaces, for which point evaluation makes sense. Note that $\sigma_{r(t)}\left(f,\left\{\left(P_{j}, \omega_{j}\right)\right\}_{j=1}^{n}\right)$ is contained in $\operatorname{Hom}_{t}\left(\mathcal{G}_{k, d}\right)$ for $r(t)=\sqrt{\left\lceil\frac{4}{k} t^{2}\right\rceil}$, cf. [14, Theorem 5] for a slightly larger function $r$. The following approximation is a consequence of [45, Proposition 5.3]:

Theorem 6 ([14]). If $\left\{\left(P_{j}^{(t)}, \omega_{j}^{(t)}\right)\right\}_{j=1}^{n_{t}}$ is a sequence of cubatures with nonnegative weights for $\operatorname{Hom}_{2 t}\left(\mathcal{G}_{k, d}\right), t=1,2, \ldots$, then, for $f \in H_{\infty}^{s}\left(\mathcal{G}_{k, d}\right)$,

$$
\left\|f-\sigma_{r(t)}\left(f,\left\{\left(P_{j}^{(t)}, \omega_{j}^{(t)}\right)\right\}_{j=1}^{n_{t}}\right)\right\|_{L_{\infty}} \lesssim t^{-s}\left(\|f\|_{L_{\infty}}+\|f\|_{H_{\infty}^{s}}\right),
$$

where $r(t)=\sqrt{\left\lceil\frac{4}{k} t^{2}\right\rceil}$.

For cubatures satisfying $n_{t} \asymp t^{k(d-k)}$, see Remark 2, (25) becomes

$$
\left\|f-\sigma_{r(t)}\left(f,\left\{\left(P_{j}^{t}, \omega_{j}^{t}\right)\right\}_{j=1}^{n_{t}}\right)\right\|_{L_{\infty}} \lesssim n_{t}^{-\frac{s}{k(d-k)}}\left(\|f\|_{L_{\infty}}+\|f\|_{H^{s}\left(L_{\infty}\right)}\right),
$$

so that we obtain error rates similiar to (18). 


\subsection{Cubatures as efficient coverings}

We have seen in the previous sections that cubatures relate to the approximation of integrals and are also useful to approximate functions from samples. Intuitively, good samplings for approximation need to cover the underlying space sufficiently well. Indeed, we shall connect cubatures with asymptotically optimal coverings.

Given any finite collection of points $\left\{P_{j}\right\}_{j=1}^{n} \subset \mathcal{G}_{k, d}$, we define the covering radius $\rho$ by

$$
\rho:=\rho\left(\left\{P_{j}\right\}_{j=1}^{n}\right):=\sup _{P \in \mathcal{G}_{k, d}} \min _{1 \leq j \leq n}\left\|P-P_{j}\right\|,
$$

where $\|\cdot\|$ denotes the Frobenius norm on the space of symmetric matrices. Let $B_{r}(P)$ denote the closed ball of radius $r$ centered at $P \in \mathcal{G}_{k, d}$. Since

$$
\mathcal{G}_{k, d}=\bigcup_{j=1}^{n} B_{\rho}\left(P_{j}\right)
$$

and $\sigma_{k, d}\left(B_{r}(P)\right) \asymp r^{k(d-k)}$, for $P \in \mathcal{G}_{k, d}$ with $0<r \leq 1$, we deduce

$$
1=\sigma_{k, d}\left(\mathcal{G}_{k, d}\right) \leq \sum_{j=1}^{n} \sigma_{k, d}\left(B_{\rho}\left(x_{j}\right)\right) \lesssim n \rho^{k(d-k)},
$$

which leads to the lower bound $n^{-\frac{1}{k(d-k)}} \lesssim \rho$. Point sequences in $\mathcal{G}_{k, d}$ that match this lower bound asymptotically in $n$ are referred to as asymptotically optimal coverings.

Theorem 7 ([13]). Any cubature sequence $\left\{\left(P_{j}^{(t)}, \omega_{j}^{(t)}\right)\right\}_{j=1}^{n_{t}}$ for $\operatorname{Hom}_{t}\left(\mathcal{G}_{k, d}\right)$ with positive weights satisfying (17) is covering asymptotically optimal, i.e., its covering radius $\rho^{(t)}$ satisfies $n^{-\frac{1}{k(d-k)}} \asymp \rho^{(t)}$.

Theorems 7 and 6 with (26) both reflect the intuition that cubature points satisfying (17) must be somewhat efficiently distributed on the underlying space.

\subsection{Cubatures for phase retrieval}

To reflect the versatility of Grassmannian cubatures, we now briefly discuss their use in phase retrieval. The problem of reconstructing vectors from phaseless magnitude measurements has attracted great attention in the recent literature, $[16,15,18,7,23]$ to mention only few. For $x \in \mathbb{R}^{d}$, the mapping

$$
\hat{x}: \mathcal{G}_{k, d} \rightarrow \mathbb{R}, \quad P \mapsto\|P x\|^{2}
$$


is a homogeneous polynomial of degree 2 , hence, contained in $\mathrm{Hom}_{2}\left(\mathcal{G}_{k, d}\right)$. Notice that $\hat{x}$ is in a one to one correspondence with the rank one matrix $x x^{\top}$ since

$$
\hat{x}(P)=x^{\top} P x=\operatorname{trace}\left(P x x^{\top}\right) .
$$

The problem of reconstructing $x x^{\top}$ from finitely many samples $\left\{\hat{x}\left(P_{j}\right)\right\}_{j=1}^{n}$, where $\left\{P_{j}\right\}_{j=1}^{n} \subset \mathcal{G}_{k, d}$, is known as the phase retrieval problem. Most publication deal with $k=1$. For $k>1$, we refer to [5, 26, 29, 27,6] and references therein.

If there are weights $\left\{\omega_{j}\right\}_{j=1}^{n}$ such that $\left\{\left(P_{j}, \omega_{j}\right)\right\}_{j=1}^{n}$ is a cubature for $\operatorname{Hom}_{2}\left(\mathcal{G}_{k, d}\right)$, then $x x^{\top}$ can be directly reconstructed via the closed formula

$$
x x^{\top}=\frac{d}{k} \sum_{j=1}^{n} \omega_{j} \hat{x}\left(P_{j}\right)\left[\frac{1}{\alpha} \sum_{j=1}^{n} \omega_{j} \hat{x}\left(P_{j}\right) P_{j}-\frac{\beta}{\alpha} I_{d}\right],
$$

where $\alpha=\frac{2 k(d-k)}{d(d+2)(d-1)}$ and $\beta=\frac{k(k d+k-2)}{d(d+2)(d-1)}$, cf. [5]. However, cubatures for $\operatorname{Hom}_{2}\left(\mathcal{G}_{k, d}\right)$ must have at least $d(d+1) / 2$ many points. Thus, the number of samples grows quadratic with the ambient dimension $d$. We are seeking reconstruction from fewer samples at the expense of replacing the closed reconstruction formula with a feasibility problem of a semidefinite program. We consider the problem

$$
\text { find } A \in \mathbb{R}_{\succeq 0}^{d \times d}, \quad \text { subject to } \operatorname{trace}\left(P_{j} A\right)=\hat{x}\left(P_{j}\right), j=1, \ldots, n,
$$

where $\mathbb{R}_{\succeq 0}^{d \times d}$ denotes the symmetric, positive semidefinite matrices in $\mathbb{R}^{d \times d}$.

Theorem $8([5])$. There are constants $c_{1}, c_{2}>0$ such that, if $n \geq c_{1} d$ and $\left\{P_{j}\right\}_{j=1}^{n} \subset \mathcal{G}_{k, d}$ are chosen independently identically distributed according to $\sigma_{k, d}$, then the matrix $x x^{\top}$ is the unique solution to (28) with probability $1-e^{-c_{2} n}$, for all $x \in \mathbb{R}^{d}$.

This theorem generalized results in $[16,15]$ from $k=1$ to $k \geq 1$. When the projectors $\left\{P_{j}\right\}_{j=1}^{n}$ are sampled from the idealized perfect cubature $\sigma_{k, d}$, then the number of samples needed grows linearly with $d$. Next, we shall find a balance between the deterministic cubatures required for (27) and the full randomness invoked by $\sigma_{k, d}$ used in Theorem 8 .

From here on, we suppose that the length $\|x\|$ is known to us. To simplify notation, we make the convention that $P_{0}=I_{d}$, hence, $\left\langle x x^{*}, P_{0}\right\rangle=$ $\operatorname{trace}\left(x x^{*}\right)=\|x\|^{2}$, consider the problem

$$
\text { find } A \in \mathbb{R}_{\succeq 0}^{d \times d}, \quad \text { such that } \operatorname{trace}\left(A P_{j}\right)=\hat{x}\left(P_{j}\right), j=0, \ldots, n \text {, }
$$

where $\left\{P_{j}\right\}_{j=1}^{n} \subset \mathcal{G}_{k, d}$ and $\hat{x}\left(I_{d}\right):=\|x\|^{2}$. Note that (29) is the feasibility problem of a semidefinite programm. For $k=1$, the following result is essentially due to [36]. The extension to $k \geq 1$ has been derived in [29]: 
Theorem 9 ([29]). Suppose that $\|x\|^{2}$ is known and that $\left\{\left(\mathcal{P}_{j}, \omega_{j}\right)\right\}_{j=1}^{n}$ is a cubature with nonnegative weights for $\operatorname{Hom}_{t}\left(\mathcal{G}_{k, d}\right), t \geq 3$. Let $\mu=\sum_{j=1}^{n} \omega_{j} \delta_{\mathcal{P}_{j}}$ denote the corresponding discrete probability measure, where $\delta_{\mathcal{P}_{j}}$ is the point measure in $\mathcal{P}_{j}$. If $\left\{P_{j}\right\}_{j=1}^{n} \subset \mathcal{G}_{k, d}$ are independently sampled from $\mu$, then with probability at least $1-e^{-\gamma}$, the rank-one matrix $x x^{*}$ is the unique solution to (29) provided that

$$
n \geq c_{1} \gamma t d^{1+2 / t} \log ^{2}(d),
$$

where $\gamma \geq 1$ is an arbitrary parameter and $c_{1}$ is a constant, which does not depend on $d$.

Hence, choosing random projectors distributed according to discrete probability measures allows us to reconstruct $x x^{\top}$ with less than $d^{2}$ many measurements.

\section{Cubatures of varying ranks}

In the previous sections, we were dealing with cubatures for Grassmannians of fixed rank. In order to allow for more flexibility, we now aim to remove this restriction, i.e., we shall investigate cubatures for unions of Grassmannians. Our present aim is to provide some elementary proofs of some of the results in [28] that were derived by the use of representation theoretic concepts.

Given a non-empty set $\mathcal{K} \subset\{1, \ldots, d-1\}$, we define the corresponding union of Grassmannians by

$$
\mathcal{G}_{\mathcal{K}, d}:=\bigcup_{k \in \mathcal{K}} \mathcal{G}_{k, d}=\left\{P \in \mathbb{R}_{\mathrm{sym}}^{d \times d}: P^{2}=P, \operatorname{trace}(P) \in \mathcal{K}\right\} .
$$

As for a single Grassmannian, the polynomials on $\mathcal{G}_{\mathcal{K}, d}$ are given by multivariate polynomials in the matrix entries of a given projector $P \in \mathcal{G}_{\mathcal{K}, d}$, i.e.,

$$
\operatorname{Pol}_{t}\left(\mathcal{G}_{\mathcal{K}, d}\right):=\left\{\left.f\right|_{\mathcal{G}_{\mathcal{K}, d}}: f \in \operatorname{Pol}_{t}\left(\mathbb{R}_{\mathrm{sym}}^{d \times d}\right)\right\} .
$$

The dimension of $\operatorname{Pol}_{t}\left(\mathcal{G}_{\mathcal{K}, d}\right)$ is an indicator of the number of points needed to obtain a cubature on $\mathcal{G}_{\mathcal{K}, d}$, cf. [21]. To compute this dimension, we shall first derive a lower bound on $\operatorname{dim}\left(\operatorname{Pol}_{t}\left(\mathcal{G}_{\mathcal{K}, d}\right)\right)$, which is relatively straight-forward:

Proposition 4. Let $\mathcal{K}=\left\{k_{i}\right\}_{i=1}^{r} \subset\{1, \ldots, d-1\}$ and $t \in \mathbb{N}_{0}$ be given such that

$$
\min \left\{k_{1}, d-k_{1}\right\} \geq \cdots \geq \min \left\{k_{r}, d-k_{r}\right\} .
$$

Then it holds

$$
\operatorname{dim}\left(\operatorname{Pol}_{t}\left(\mathcal{G}_{\mathcal{K}, d}\right)\right) \geq \sum_{i=1}^{s} \operatorname{dim}\left(\operatorname{Pol}_{t-i+1}\left(\mathcal{G}_{k_{i}, d}\right)\right), \quad s:=\min \{t+1,|\mathcal{K}|\} .
$$


Note that the dimension of each $\operatorname{Pol}_{t-i+1}\left(\mathcal{G}_{k_{i}, d}\right)$ is known, i.e.,

$$
\operatorname{dim}\left(\operatorname{Pol}_{t}\left(\mathcal{G}_{k, d}\right)\right)=\sum_{\substack{|\pi| \leq t, \ell(\pi) \leq \min \{k, d-k\}}} \mathcal{D}(d, 2 \pi),
$$

where

$$
\mathcal{D}(d, \pi)=\prod_{1 \leq i<j \leq \frac{d}{2}} \frac{\left(l_{i}+l_{j}\right)\left(l_{i}-l_{j}\right)}{(j-i)(d-i-j)} \cdot \begin{cases}\prod_{1 \leq i \leq \frac{d}{2}} \frac{2 l_{i}}{d-2 i}, & d \text { odd, } \\ 2, & d \text { even and } \pi_{\left\lfloor\frac{d}{2}\right\rfloor}>0, \\ 1, & d \text { even and } \pi_{\left\lfloor\frac{d}{2}\right\rfloor}=0,\end{cases}
$$

with $l_{i}:=\frac{d}{2}+\pi_{i}-i$, for $1 \leq i \leq \frac{d}{2}$, cf. [33, Formulas (24.29) and (24.41)] and $[2,3]$. Thus, $(33)$ is an explicit lower bound on the dimension of $\mathrm{Pol}_{t}\left(\mathcal{G}_{\mathcal{K}, d}\right)$.

Proof (of Proposition 4). We will show that the lower bound (33) is valid for any ordering of the indices $k_{1}, \ldots, k_{r}$. In particular it holds for the ordering specified in (32), which maximizes the right hand side over all such lower bounds.

For $t=0$ or $r=1$, the sum in (33) reduces to a single term, so that the lower bound indeed holds. For fixed $t \geq 1$, we verify the general case by induction over $r$, where we proceed from $r-1$ to $r$ with $r \geq 2$.

Choose $\left\{f_{i}\right\}_{i=1}^{m} \subset \operatorname{Pol}_{t}\left(\mathbb{R}_{\mathrm{sym}}^{d \times d}\right)$ and $\left\{g_{j}\right\}_{j=1}^{n} \subset \operatorname{Pol}_{t-1}\left(\mathbb{R}_{\mathrm{sym}}^{d \times d}\right)$ such that $\left\{\left.f_{i}\right|_{\mathcal{G}_{k_{1}, d}}\right\}_{i=1}^{m}$ and $\left\{\left.g_{j}\right|_{\left.\mathcal{G}_{\mathcal{K} \backslash\left\{k_{1}\right\}, d}\right\}_{j=1}^{n}}\right.$ are bases for the spaces $\operatorname{Pol}_{t}\left(\mathcal{G}_{k_{1}, d}\right)$ and $\mathrm{Pol}_{t-1}\left(\mathcal{G}_{\mathcal{K} \backslash\left\{k_{1}\right\}, d}\right)$, respectively. We infer that any linear combination

$$
h:=\left.\sum_{i=1}^{m} \alpha_{i} f_{i}\right|_{\mathcal{G}_{\mathcal{K}, d}}+\left.\sum_{j=1}^{n} \beta_{j}\left(\operatorname{Tr}(\cdot)-k_{1}\right) g_{j}\right|_{\mathcal{G}_{\mathcal{K}, d}}
$$

is contained in $\operatorname{Pol}_{t}\left(\mathcal{G}_{\mathcal{K}, d}\right)$. Suppose now that $h$ vanishes on $\mathcal{G}_{\mathcal{K}, d}$. In particular, $h$ vanishes on $\mathcal{G}_{k_{1}, d}$, so that $\alpha_{i}=0, i=1, \ldots, m$. Vanishing on $\mathcal{G}_{\mathcal{K} \backslash\left\{k_{1}\right\}, d}$ implies $\beta_{j}=0, j=1, \ldots, n$. Hence, the function system

$$
\left\{\left.f_{i}\right|_{\mathcal{G}_{\mathcal{K}, d}}\right\}_{i=1}^{m} \cup\left\{\left.\left(\operatorname{Tr}(\cdot)-k_{1}\right) g_{j}\right|_{\mathcal{G}_{\mathcal{K}, d}}\right\}_{j=1}^{n}
$$

is linearly independent in $\operatorname{Pol}_{t}\left(\mathcal{G}_{\mathcal{K}, d}\right)$. By using $s-1=\min \{t, r-1\}$, we infer by the induction hypothesis 


$$
\begin{aligned}
\operatorname{dim}\left(\operatorname{Pol}_{t}\left(\mathcal{G}_{\mathcal{K}, d}\right)\right) & \geq \operatorname{dim}\left(\operatorname{Pol}_{t}\left(\mathcal{G}_{k_{1}, d}\right)\right)+\operatorname{dim}\left(\operatorname{Pol}_{t-1}\left(\mathcal{G}_{\mathcal{K} \backslash\left\{k_{1}\right\}, d}\right)\right) \\
& \geq \operatorname{dim}\left(\operatorname{Pol}_{t}\left(\mathcal{G}_{k_{1}, d}\right)\right)+\sum_{i=1}^{s-1)} \operatorname{dim}\left(\operatorname{Pol}_{(t-1)-i+1}\left(\mathcal{G}_{k_{i+1}, d}\right)\right) \\
& =\operatorname{dim}\left(\operatorname{Pol}_{t}\left(\mathcal{G}_{k_{1}, d}\right)\right)+\sum_{i=2}^{s} \operatorname{dim}\left(\operatorname{Pol}_{t-i+1}\left(\mathcal{G}_{k_{i}, d}\right)\right) \\
& =\sum_{i=1}^{s} \operatorname{dim}\left(\operatorname{Pol}_{t-i+1}\left(\mathcal{G}_{k_{i}, d}\right)\right)
\end{aligned}
$$

which proves the lower bound (33).

In case $\mathcal{K}=\{k, d-k\}$, we can verify that the lower bound is matched by elementary methods:

Proposition 5. Let $1 \leq k \leq d-1$ with $k \neq \frac{d}{2}$ and $t \geq 1$. Then it holds

$$
\operatorname{Pol}_{t}\left(\mathcal{G}_{k, d} \cup \mathcal{G}_{d-k, d}\right) \cong \operatorname{Pol}_{t}\left(\mathcal{G}_{k, d}\right) \oplus \operatorname{Pol}_{t-1}\left(\mathcal{G}_{d-k, d}\right)
$$

Proof. We consider the restriction mapping

$$
\left.\right|_{\mathcal{G}_{k, d}}: \operatorname{Pol}_{t}\left(\mathcal{G}_{k, d} \cup \mathcal{G}_{d-k, d}\right) \longrightarrow \operatorname{Pol}_{t}\left(\mathcal{G}_{k, d}\right),\left.\quad f \mapsto f\right|_{\mathcal{G}_{k, d}}
$$

and shall verify that the dimension of its nullspace satisfies

$$
\operatorname{null}\left(\left.\right|_{\mathcal{G}_{k, d}}\right)=(\operatorname{trace}(\cdot)-k) \operatorname{Pol}_{t-1}\left(\mathcal{G}_{k, d} \cup \mathcal{G}_{d-k, d}\right)
$$

Since $\left.\right|_{\mathcal{G}_{k, d}}$ is onto and $(\operatorname{trace}(\cdot)-k) \mathrm{Pol}_{t-1}\left(\mathcal{G}_{k, d} \cup \mathcal{G}_{d-k, d}\right)$ is equivalent to $\mathrm{Pol}_{t-1}\left(\mathcal{G}_{d-k, d}\right)$, this would imply $(36)$.

It is obvious that the right-hand-side in (37) is contained in null $\left(\left.\right|_{\mathcal{G}_{k, d}}\right)$. The latter can also be deduced from the lower bounds (33). For the reverse set inclusion, let $f \in \operatorname{null}\left(\left.\right|_{\mathcal{G}_{k, d}}\right)$. We must now check that $\left.f\right|_{\mathcal{G}_{d-k, d}} \in$ $\operatorname{Pol}_{t-1}\left(\mathcal{G}_{d-k, d}\right)$.

To proceed let us denote $n:=\operatorname{dim}\left(\operatorname{Pol}_{t}\left(\mathcal{G}_{k, d} \cup \mathcal{G}_{d-k, d}\right)\right)$. According to [28], see also [4], there are $\left\{X_{j}\right\}_{j=1}^{n} \subset \mathcal{G}_{k, d} \cup \mathcal{G}_{d-k, d}$ and $\left\{c_{j}\right\}_{j=1}^{n} \subset \mathbb{R}$ such that

$$
f(P)=\left.\sum_{j=1}^{n} c_{j} \operatorname{trace}\left(X_{j} P\right)^{t}\right|_{\mathcal{G}_{k, d} \cup \mathcal{G}_{d-k, d}}, \quad P \in \mathcal{G}_{k, d} \cup \mathcal{G}_{d-k, d} .
$$

By applying the binomial formula, we observe that

$$
f+(-1)^{t+1} f\left(I_{d}-\cdot\right) \in \operatorname{Pol}_{t-1}\left(\mathcal{G}_{k, d} \cup \mathcal{G}_{d-k, d}\right) .
$$

Therefore, the assumption $\left.f\right|_{\mathcal{G}_{k, d}} \equiv 0$ implies that $\left.f(I-\cdot)\right|_{\mathcal{G}_{k, d}} \in \operatorname{Pol}_{t-1}\left(\mathcal{G}_{k, d}\right)$. Since $f \mapsto f(I-\cdot)$ is an isomorphism between $\operatorname{Pol}_{t-1}\left(\mathcal{G}_{k, d}\right)$ and $\operatorname{Pol}_{t-1}\left(\mathcal{G}_{d-k, d}\right)$, we derive $\left.f\right|_{\mathcal{G}_{d-k, d}} \in \operatorname{Pol}_{t-1}\left(\mathcal{G}_{d-k, d}\right)$. Thus, we have verified (37), which concludes the proof. 
Proposition 5 shows that, for $\mathcal{K}=\{k, d-k\}$, the inequality in Proposition 4 is an equality. It has been proved in [28] that there also holds equality in the general situation:

Theorem 10 ([28]). Let $\mathcal{K}=\left\{k_{i}\right\}_{i=1}^{r} \subset\{1, \ldots, d-1\}$ and $t \in \mathbb{N}_{0}$ be given such that

$$
\min \left\{k_{1}, d-k_{1}\right\} \geq \cdots \geq \min \left\{k_{r}, d-k_{r}\right\} .
$$

Then it holds

$$
\operatorname{Pol}_{t}\left(\mathcal{G}_{\mathcal{K}, d}\right) \cong \bigoplus_{i=1}^{s} \operatorname{Pol}_{t-i+1}\left(\mathcal{G}_{k_{i}, d}\right), \quad s:=\min \{t+1,|\mathcal{K}|\}
$$

Compared to our elementary proofs of Propositions 4 and 5, the proof of Theorem 10 presented in [28] is much more involved. It makes use of representation theoretic concepts in combination with orthogonally invariant reproducing kernels.

Note that Theorem 10 reveals that each $f \in \operatorname{Pol}_{t}\left(\mathcal{G}_{\mathcal{K}, d}\right)$ vanishing on $\operatorname{Pol}_{t}\left(\mathcal{G}_{k_{1}, d}\right)$ must contain a factor $\left.\left(\operatorname{Tr}(\cdot)-k_{1}\right)\right|_{\mathcal{G}_{\mathcal{K}, d}}$, i.e., the restriction mapping $\left.\right|_{\mathcal{G}_{k_{1}, d}}$ from $\operatorname{Pol}_{t}\left(\mathcal{G}_{\mathcal{K}, d}\right)$ to $\operatorname{Pol}_{t}\left(\mathcal{G}_{k_{1}, d}\right)$, for $t \geq 1$, satisfies

$$
\operatorname{null}\left(\left.\right|_{\mathcal{G}_{k_{1}, d}}\right)=\left(\operatorname{Tr}(\cdot)-k_{1}\right) \operatorname{Pol}_{t-1}\left(\mathcal{G}_{\mathcal{K}, d}\right) .
$$

Understanding the structure of polynomials on $\mathcal{G}_{\mathcal{K}, d}$ is one of the key ingredients to apply the concept of cubatures in the areas of the previous sections. While we now better understand the space $\operatorname{Pol}_{t}\left(\mathcal{G}_{\mathcal{K}, d}\right)$, there is still work to do in order to approximate integrals and functions defined on unions of Grassmannians $\mathcal{G}_{\mathcal{K}, d}$, to deal with phase retrieval problems when magnitude is measured in subspaces of varying dimensions, and to derive high dimensional moment reconstructions from marginal moments of varying low dimensions. This shall be addressed in future work.

Acknowledgements Thomas Peter was funded by the German Academic Exchange Service (DAAD) through P.R.I.M.E. 57338904. All authors have been supported by the Vienna Science and Technology Fund (WWTF) through project VRG12-009.

\section{References}

1. D. Achlioptas, Database-friendly random projections: Johnson-lindenstrauss with binary coins, Journal of Computer and System Sciences 66 (2003), no. 4, 671-687.

2. C. Bachoc, E. Bannai, and R. Coulangeon, Codes and designs in Grassmannian spaces, Discrete Mathematics 277 (2004), 15-28.

3. C. Bachoc, R. Coulangeon, and G. Nebe, Designs in Grassmannian spaces and lattices, J. Algebraic Combinatorics 16 (2002), 5-19.

4. C. Bachoc and M. Ehler, Tight p-fusion frames, Appl. Comput. Harmon. Anal. 35 (2013), no. 1, 1-15. 
5. Signal reconstruction from the magnitude of subspace components, IEEE Trans. Inform. Theory 61 (2015), no. 7, 1-13.

6. S. Bahmanpour, J. Cahill, P. G. Casazza, J. Jasper, and L. M. Woodland, Phase retrieval and norm retrieval, Trends in harmonic analysis and its applications, Contemp. Math. 650 (2015), 3-14.

7. A. S. Bandeira, J. Cahill, D. G. Mixon, and A. A. Nelson, Saving phase: Injectivity and stability for phase retrieval, Appl. Comput. Harmon. Anal. 37 (2014), no. 1, $106-125$.

8. G. Baron, de prony, "essai expérimental et analytique sur les lois de la dilatabilité des fluides élastique et sur celles de la force expansive de la vapeur de l'eau et de la vapeur de l'alkool, à différentes températures", Journal de l'École Polytechnique 1 (1795), no. 2, 24-76.

9. D. J. Bates, J. D. Hauenstein, A. J. Bellommese, and C. W. Wampler, Numerically solving polynomial systems with Bertini, vol. 25, SIAM, 2013.

10. B. Bodman, M. Ehler, and M. Gräf, Moment fusion: From low to highdimensional moments without magic, arXiv (2015).

11. L. Brandolini, C. Choirat, L. Colzani, G. Gigante, R. Seri, and G. Travaglini, Quadrature rules and distribution of points on manifolds, Annali della Scuola Normale Superiore di Pisa - Classe di Scienze XIII (2014), no. 4, 889-923.

12. J. Brauchart, E. Saff, I. H. Sloan, and R. Womersley, QMC designs: Optimal order quasi Monte Carlo integration schemes on the sphere, Math. Comp. $\mathbf{8 3}$ (2014), 2821-2851.

13. A. Breger, M. Ehler, and M. Gräf, Points on manifolds with asymptotically optimal covering radius, arXiv (2016).

14. A. Breger, M. Ehler, and M. Gräf, Quasi Monte Carlo integration and kernelbased function approximation on Grassmannians, Frames and Other Bases in Abstract and Function Spaces: Novel Methods in Harmonic Analysis, vol. 1, Birkhauser/Springer, 2017.

15. E. J. Candès and X. Li., Solving quadratic equations via PhaseLift when there are about as many equations as unknowns, Foundations of Computational Mathematics 14 (2014), 1017-1026.

16. E. J. Candès, T. Strohmer, and V. Voroninski, PhaseLift: Exact and stable signal recovery from magnitude measurements via convex programming, Communications on Pure and Applied Mathematics, DOI:10.1002/cpa.21432 66 (2013), no. 8, 1241-1274.

17. Y. Chikuse, Statistics on special manifolds, Lecture Notes in Statistics, Springer, New York, 2003.

18. A. Conca, D. Edidin, M. Hering, and C. Vinzant, An algebraic characterization of injectivity in phase retrieval, Appl. Comput. Harmon. Anal 38 (2015), no. 2, 346-356.

19. S. Dasgupta and A. Gupta, An elementary proof of a theorem of Johnson and Lindenstrauss, Random Structures \& Algorithms 22 (2003), no. 1, 60-65.

20. C. de Boor, K. Hölling, and S. D. Riemenschneider, Box Splines, Springer, New York, 1993.

21. P. de la Harpe and C. Pache, Cubature formulas, geometrical designs, reproducing kernels, and Markov operators, Infinite groups: geometric, combinatorial and dynamical aspects (Basel), vol. 248, Birkhäuser, 2005, pp. 219-267.

22. P. Delsarte, J. M. Goethals, and J. J. Seidel, Spherical codes and designs, Geom. Dedicata 6 (1977), 363-388.

23. L. Demanet and P. Hand, Stable optimizationless recovery from phaseless linear measurements, J. Fourier Anal. Appl. 20 (2014), 199-221.

24. R. A. DeVore and G. G. Lorentz, Constructive Approximation, Springer-Verlag, 1993. 
25. I. Dumitriu, A. Edelman, and G. Shuman, MOPS: Multivariate orthogonal polynomials (symbolically), Journal of Symbolic Computation 42 (2007), no. 6, 587620.

26. D. Edidin, Projections and phase retrieval, Appl. Comput. Harmon. Anal 42 (2017), no. 2, 350-359.

27. M. Ehler, M. Fornasier, and J. Sigl, Quasi-linear compressed sensing, SIAM Multiscale Modeling and Simulation 12 (2014), no. 2, 725-754.

28. M. Ehler and M. Gräf, Reproducing kernels for the irreducible components of polynomial spaces on unions of Grassmannians, arXiv (2017).

29. M. Ehler, F. Kiraly, and M. Gräf, Phase retrieval using cubatures of positive semidefinite matrices, Waves, Wavelets and Fractals - Adv. Anal. 1 (2015), no. 1, $32-50$.

30. H. Engels, Numerical quadrature and cubature, Computational mathematics and applications, Academic Press, London, 1980.

31. U. Etayo, J. Marzo, and J. Ortega-Cerda, Asymptotically optimal designs on compact algebraic manifolds, arXiv (2016)

32. F. Filbir and H. N. Mhaskar, A quadrature formula for diffusion polynomials corresponding to a generalized heat kernel, J. Fourier Anal. Appl. 16 (2010), no. $5,629-657$.

33. W. Fulton and J. Harris, Representation theory, a first course, Springer, 1991.

34. D. Geller and I. Z. Pesenson, Band-limited localized Parseval frames and Besov spaces on compact homogeneous manifolds, J. Geom. Anal. 21 (2011), no. 2, 334-371.

35. M. Gräf, Efficient algorithms for the computation of optimal quadrature points on Riemannian manifolds, Universitätsverlag Chemnitz, 2013.

36. D. Gross, F. Krahmer, and R. Kueng, A partial derandomization of PhaseLift using spherical designs, J. Fourier Anal. Appl. 21 (2015), no. 2, 229-266.

37. K. Gross and D. St. P. Richards, Special functions of matrix argument. I: Algebraic induction, zonal polynomials and hypergeometric functions, Trans. Amer. Math. Soc. 301 (1987), 781-811.

38. M. T. Harandi, C. Sanderson, S. Shirazi, and B. C. Lovell, Kernel analysis on Grassmann manifolds for action recognition, Pattern Recognition Letters 34 (2013), no. 15, 1906-1915.

39. S. G. Hoggar, t-designs in projective spaces, Europ. J. Combinatorics 3 (1982), $233-254$.

40. A. T. James and A. G. Constantine, Generalized Jacobi polynomials as spherical functions of the Grassmann manifold, Proc. London Math. Soc. 29 (1974), no. 3, 174-192.

41. H. König, Cubature formulas on spheres, Adv. Multivar. Approx. Math. Res. 107 (1999), 201-211.

42. S. Kunis, T. Peter, T. Römer, and U. von der Ohe, A multivariate generalization of Prony's method, Lin. Alg. Appl. 490 (2016), 31-47.

43. M. Maggioni and H. N. Mhaskar, Diffusion polynomial frames on metric measure spaces, Appl. Comput. Harmon. Anal. 24 (2008), no. 3, 329-353.

44. J. Matousek, On variants of the Johnson-Lindenstrauss lemma, Random Structures \& Algorithms 33 (2008), no. 2, 142-156.

45. H. N. Mhaskar, Eignets for function approximation on manifolds, Appl. Comput. Harmon. Anal. 29 (2010), 63-87.

46. R. J. Muirhead, Aspects of multivariate statistical theory, John Wiley \& Sons, New York, 1982.

47. A. Neumaier and J. J. Seidel, Discrete measures for spherical designs, eutactic stars and lattices, Indag. Math. 91 (1988), no. 3, 321-334.

48. E. Novak and H. Wozniakowski, Tractability of Multivariate Problems. Volume II, EMS Tracts in Mathematics, vol. 12, EMS Publishing House, Zürich, 2010. 
49. I. Z. Pesenson and D. Geller, Cubature formulas and discrete fourier transform on compact manifolds, From Fourier Analysis and Number Theory to Radon Transforms and Geometry, vol. 28, 2012, pp. 431-453.

50. T. Peter and G. Plonka, A generalized Prony method for reconstruction of sparse sums of eigenfunctions of linear operators, Inverse Problems 29 (2013), no. 2, 025001.

51. G. Plonka and M. Tasche, Prony methods for recovery of structured functions, GAMM-Mitteilungen 37 (2014), no. 2, 239-258.

52. A. Reznikov and E. B. Saff, The covering radius of randomly distributed points on a manifold, International Mathematics Research Notices rnv342 (2015).

53. R. Roy, A. Paulraj, and T. Kailath, ESPRIT - A subspace rotation approach to estimation of parameters of cisoids in noise, IEEE Trans. Acoust., Speech, Signal Process. 34 (1986), no. 5, 1340-1342.

54. R. Schmidt, Multiple emitter location and signal parameter estimation, IEEE Trans. Antennas Propag. 34 (1986), no. 3, 276-280.

55. P. Turaga, A. Veeraraghavan, A. Srivastava, and R. Chellappa, Statistical computations on Grassmann and Stiefel manifolds for image and video-based recognition, IEEE Trans. Pattern Anal. Mach. Intell. 33 (2011), no. 11, 2273-2286.

56. M. Vetterli, P. Marziliano, and T. Blu, Sampling signals with finite rate of innovation, IEEE Trans. Signal Process. 50 (2002), no. 6, 1417-1428.

57. I. B. Yaacov, A multivariate version of the vandermonde determinant identity, arXiv:1405.0993 (2014). 